\title{
Design of a Legendre-Polynomial-Based Orthogonal Pulse Generator for Ultra- Wideband Communications
}

\author{
Chih-Yung Tsai* and Shyh-Kang Jeng \\ Graduate Institute of Communication Engineering and \\ Department of Electrical Engineering \\ National Taiwan University \\ Taipei, Taiwan \\ Email: d89006@ew.ee.ntu.edu.tw \\ skjeng@ew.ee.ntu.edu.tw
}

\begin{abstract}
We propose a novel circuit for generation of Legendre-polynomial-based pulses for use in impulse radio ultra wideband (UWB) communications. Both parallel and serial generation of pulses of different order can be developed. Pulses are produced by applying Bonnet's recursion formula which can generate the Legendre polynomial functions of successive orders. This class of functions yield orthogonal pulses and have a constant pulse width regardless of the pulse order. This nice property can eliminate inter-symbol interference (ISI) such that these pulses are suitable for use in pulse position modulation (PPM) ultra wideband communication systems.
\end{abstract}

\section{Introduction}

One of the essential parts in digital communications is the representation of a message symbol by an analog waveform for transmission through a channel. In UWB technology the analog waveform is a simple pulse which transmitted without up conversion. It is a base-band technique, which thus reduce the complexity and cost of the transceiver and makes it attractive as a wireless communication method. In contrast with the conventional communication systems using the sinusoidal carriers, UWB information is carried in very short pulse, which covers an extremely wide spectrum bandwidth and provides high data rates for wireless communication $[1,2,3]$.

The typical and most-used pulses by now are the Gaussian pulse and its derivatives due to some nice properties described in [1]. The Gaussian pulse generators designed by transmission-line technology $[2,3]$ and by CMOS technology [4] which consist of simple structures are already available by some electronic vendors. But according to some researches, they are not the most suitable pulses for UWB communication under the considerations of power spectral density (PSD), power consuming efficiency and the characteristics of radio frequency components used in the front-end circuits of UWB communication systems $[5,6,7]$. The most important restriction for UWB applications is the equivalent isotropically radiated power (EIRP) mask defined by the Federal Communication Commission (FCC).

Starting from the spectral control techniques, one can lead to the mathematical model of prolate spheroidal wave functions (PSWF) $[7,8]$. By using the pre-defined PSD mask as the filter design specification, we can find that the most suitable pulses are the PSWF solutions to such a mask. And the kernel of such solutions are the Legendre polynomial functions [8,9]. Hence, the design of Legendre-polynomial pulse generator for each order 
is the first step toward the generation of the best pulse shapes applied in UWB communication systems. In this paper, we design this special pulse generator circuit by block diagrams, and each diagram is simulated with Simulink tools in MATLAB. From the output data of time-domain waveforms and spectral-domain distributions, we can recognize that the different order of Legendre polynomial pulses will fit different band with less signal distortion and power waste. Moreover, the solution pulses of PSWF can be generated by suitable combinations of some different orders of Legendre polynomial pulses with appropriate weightings.

\section{Theoretical Analysis}

Given a desired frequency mask $H(f)$, we can obtain its corresponding impulse response $h(t)$. For the pulse signal $\psi(t)$ that is time-limited to $T_{m}$ (impulse pulse period) seconds while exhibiting minimal distortion, maximal power efficiency, and the widest bandwidth (BW), the corresponding spectral distribution $\Psi(f)$ with bandwidth $\Omega$ should reside in $H(f)$ with the least square difference. For the FCC EIRP mask or the band definitions of direct-sequence UWB (DS-UWB), $h(t)$ can be expressed by sinc functions. The optimal pulse signal $\psi(t)$ which meets those requirements then satisfies

$$
\int_{-T_{m} / 2}^{T_{m} / 2} \psi(v) \frac{\sin \Omega(t-v)}{\pi(t-v)} d v=\lambda \psi(v)
$$

Its solution can be expressed as [8]

$$
\psi_{l}(t)=\psi_{l}\left(\Omega, T_{m}, t\right)=\frac{\left[2 \lambda_{l}(c) / T_{m}\right]^{\frac{1}{2}} S_{0 l}^{1}\left(c, 2 t / T_{m}\right)}{\left\{\int_{1}^{1}\left[S_{0 l}^{1}(c, v)\right]^{2} d v\right\}^{\frac{1}{2}}}
$$

where

$$
S_{0 l}^{1}(c, t)= \begin{cases}\sum_{n=0,2, \cdots}^{\infty} d_{n}(c) P_{n}(c, t) ; l: \text { even } \\ \sum_{n=1,3, \cdots}^{\infty} d_{n}(c) P_{n}(c, t) ; l: \text { odd }\end{cases}
$$

and $c=\Omega T_{m} / 2, \quad P_{n}(c, t)$ is a Legendre polynomial, $d_{n}(c)$ satisfy some complex recurrence relation which represent weightings for $P_{n}(c, t)$.

As we all know that $P_{n}(x)$ can be generated by Bonnet's recursion:

$$
P_{n+1}(x)=\frac{2 n+1}{n+1} x P_{n}(x)-\frac{n}{n+1} P_{n-1}(x) \Rightarrow P_{n+1}=k_{b n} \cdot x \cdot P_{n}-k_{a n} \cdot P_{n-1}
$$

Here comes to our design formula for the Legendre-polynomial pulse generator (LPPG). 


\section{Circuit Design and Results}

The circuit of LPPG consists of two parts. The coefficients $k_{b n}$ and $k_{a n}$ in (4) are designed as the upper part in Fig. 1. The inputs are the initial order value $n$ and a DC bias level 1. For the whole circuit, the inputs include $P_{0}$ and $P_{1}$ as well as a signal amplitude varies from -1 to +1 during a period of time. Obviously, each stage is identical such that we can transform the parallel LPPG in Fig. 1 to the serial one in Fig. 2. If we combine a serial LPPG with an m-stages parallel one via some switches, we can produce $\mathrm{m}$ successive Legendre orthogonal pulses. In the demo results in Fig. 3, we set the period equals the amplitude variation, and at each stage $n$ the output is $P_{n+1}$. It is clear that the time-width is constant regardless of the pulse order. In Fig. 4, we see that the main lobe of PSD shifts to higher frequency band as pulse order increases. So, for specific PSD mask, we can use the moment method to find the most suitable combination of orthogonal legendre pulses to meet the requirements.

\section{Conclusions}

In this paper, we have proposed a novel circuit diagram to implement the Legendre polynomial pulses based on Bonnet's recursion formula which can generate multiple orthogonal pulses simultaneously. There are several applications. First, we can combine some specific pulses to achieve a specified PSD mask with the least filtering and thus keep the signal integrity as well as avoid power waste. Second, by constructing a set of orthogonal pulses, an M-ary signaling set can be constructed, allowing higher data rates. Alternatively, in a multi-user system these pulses can be assigned to different users. Third, according to theoretical analysis, the solution of PSWF is one of the best pulse shapes for UWB applications, and the key point to apply such pulses is to design the Legendrepolynomial pulse generator.

\section{References}

[1] X. Chen and S. Kiaei, "Monocycle shapes for ultra wideband system", IEEE ISCAS 2002., Vol.1 , 26-29 May 2002

[2] J. S. Lee and C. Nguyen, "Novel Low-Cost Ultra-Wideband, Ultra-Short-Pulse Transmitter with MESFET Impulse-Shaping Circuitry for Reduced Distortion and Improved Pulse Repetition Rate", IEEE Microwave and Wireless Components Letters, Vol.11, No.5, May, 2001

[3] J. Han and C. Nguyen, "A New Ultra-Wideband, Ultra-short Monocycle Pulse Generator With Reduced Ringing", IEEE Microwave and Wireless Components Letters, Vol.12, No.6, Jun. 2002

[4] H. Kim, D. Park, and Y. Joo, "Design of CMOS Scholtz's Monocycle Pulse Generator", IEEE, 2003

[5] C. A. Corral, S. Sibecas, S. Emami, and G. Stratis, "Pulse spectrum optimization for ultra-wideband communication," IEEE Conference on UWBST 2002, Digest of Papers, pp.31-35, 21-23 May 2002

[6] David M. Pozar, "Waveform Optimizations for Ultrawideband Radio Systems," IEEE Transactions on Antennas and Propagation, Vol. 51, No. 9, Sep., 2003

[7] B. Parr, B. Cho, K. Wallace, and Z. Ding, "A Novel Ultra-Wideband Pulse Design Algorithm", IEEE Communication Letters, Vol.7, No.5, May,2003 
[8] R. S. Dilmaghani, M. Ghavami, B. Allen, and H. Aghvami, "Novel UWB pulse shaping using prolate spheroidal wave functions", IEEE Proceedings on PIMRC 2003, Vol.1, Sep. 2003

[9] Z. Lin, R. W. McCallum, and H. Wang, "Computation and performance of the prolate-spheroidal wave function window in spectral estimation", IEEE International Conference on ICASSP 1996, Vol.5, May. 1996

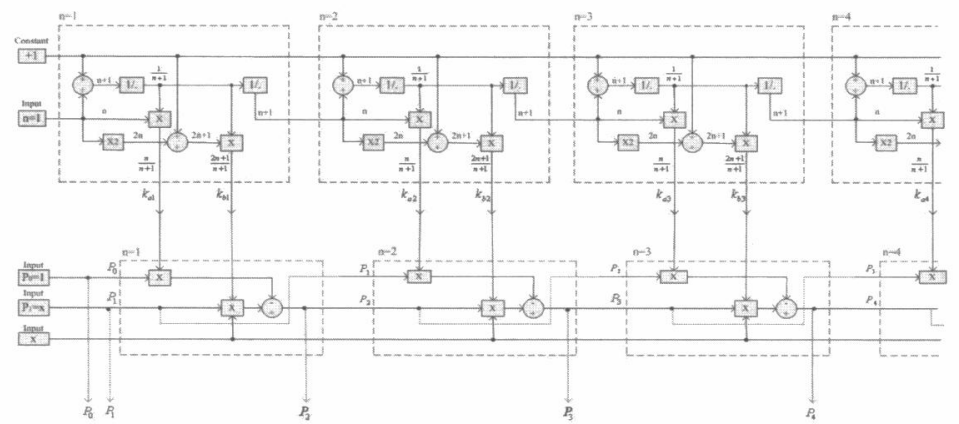

Fig. 1 Parallel circuit diagram of Legendre-polynomial pulse generator (LPPG).

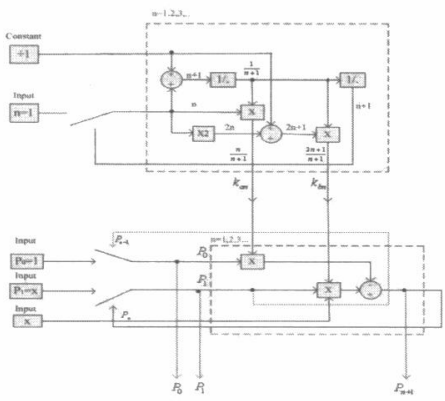

Fig. 2 Serial circuit diagram of LPPG.

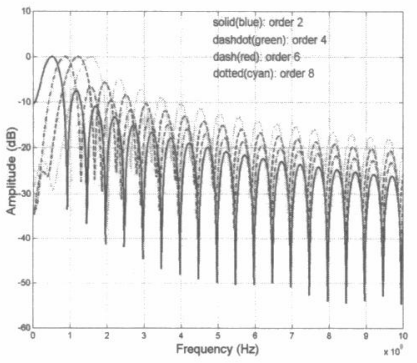

Fig. 4 PSD of LPPG output waveforms.

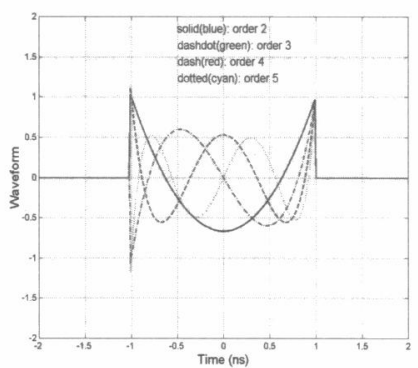

Fig. 3 Output waveforms of LPPG. 